ISSN electrónico: 2445-1355

DOI: https://dx.doi.org/10.14201/fj202052714

\title{
PATRÓN EPIDEMIOLÓGICO DEL USO DE BENZODIAZEPINAS
}

\section{Epidemiological Pattern of Benzodiazepine Use}

Abel BARRIOS; Ramona MATEOS

Departamento de Ciencias Biomédicas y del Diagnóstico. Área Medicina Preventiva y Salud Pública. Facultad de Medicina. Universidad de Salamanca

Correo-e: abelbarry60@usal.es; rmateos@usal.es

RESUMEN: El consumo de benzodiazepinas ha aumentado desde su incursión en terapéutica en la década de los noventa hasta hoy. Se trata, además, de uno de los grupos farmacológicos más prescritos hoy día en España.

Las mujeres de avanzada edad son las consumidoras mayoritarias y los principios activos más consumidos son el Alprazolam y el Lormetazepam. Se prescriben principalmente para tratamientos crónicos, como ansiolíticos y en Atención Primaria. Pese a que una gran mayoría no reconoce tener efectos adversos, los más abundantes en la población son dependencia y tolerancia y los menos frecuentes somnolencia y sedación excesiva.

Teniendo todo esto en cuenta es posible mejorar el uso de benzodiazepinas en el tiempo de uso adecuándolo al que indica la ficha téncica de medicamento, acompañando esto de medidas sociales ajustadas a cada paciente.

Palabras clave: Benzodiazepinas; consumo; indicación; ansiolítico.

ABSTRACT: The consumption of benzodiazepines has increased since its incursion in therapy in the nineties until today. It is also one of the most prescribed pharmacological groups in Spain today.

Older women are the majority consumers and the most consumed active drugs are Alprazolam and Lormetazepam. They are prescribed primarily for chronic treatments, such as anxiolytics and in Primary Care. 
Although a large majority do not recognize having adverse effects, the most abundant in the population are dependence and tolerance and the less frequent drowsiness and excessive sedation.

Taking all this into account, it is possible to improve the use of benzodiazepines in the time of use, adapting it to the one indicated in the technical data sheet, accompanying this with social measures adjusted to each patient.

Keywords: Benzodiazepines; consume; indication; ansiolitic.

\section{INTRODUCCIÓN}

Las benzodiazepinas poseen acción ansiolítica, miorrelajante, anticonvulsivante e hipnótica principalmente. Realizan todas estas acciones debido a que se fijan de manera específica a una parte concreta del complejo molecular del receptor GABAA; modifican la disposición estructural tridimensional, facilitando la apertura del receptor. Ejercen, por tanto, una modulación alostérica que aumenta la influencia GABAérgica (Lorenzo et al., 2009; Flórez et al., 2014; Goodman, Gilman, 2007).

Las benzodiazepinas son uno de los grupos farmacológicos más prescritos en los países desarrollados. (Hollingworth, Siskind, 2010; Lagnaoui et al., 2004). Diversos estudios (Rayón et al., 1997; García et al., 2004) han mostrado que el consumo aumentó de manera considerable y sostenida en España en los años 90. Otros análisis sugieren que España estaría situada por encima de la media europea en el consumo de ansiolíticos (Simó, 2012; Khomg et al., 2012). En una encuesta de abuso de drogas se ha estimado que la prevalencia de uso de hipnótico-sedantes en España durante el año 2011 fue del 11,4 \% en la población general (Ministerio de Sanidad, 2013). El consumo se incrementó en un 57,4 \% en el periodo $2000-$ 2012 (AEMPS, 2014).

Los hipnótico-sedantes más consumidos en el año 2012 en España fueron Lormetazepam y Zolpidem con 20,3 y 8,0 DHD respectivamente. En cambio, Triazolam, Flunitrazepam, Quazepam, Loprazolam y Clometiazol experimentaron un descenso en el consumo. Como ansiolíticos los más consumidos fueron Lorazepam y Alprazolam (22,0 y 16,7 DHD respectivamente). El incremento más importante se registró para Lorazepam e Hidroxizina ( $87,6 \%$ y 56,6 \% respectivamente) seguidos de Diazepam y Alprazolam (52,2 \% y 46,4 \% respectivamente). Se observó un descenso en el uso de Pinazepam, Bentazepam y Clotiazepam (AEMPS, 2014). 


\section{Objetivos}

\subsection{Objetivo general}

Conocer el patrón epidemiológico del consumo de Benzodiazepinas independientemente de la indicación para la que sea utilizada.

\subsection{Objetivos específicos}

1. Analizar el perfil del paciente según variables epidemiológicas de persona: edad, sexo, nivel de estudios, profesión o situación laboral.

1. Identificar las benzodiazepinas más prescritas.

2. Conocer el origen de la prescripción (Atención Primaria o Especializada).

3. Estudiar la indicación para la que se utiliza la benzodiazepina.

4. Evaluar la cronicidad del tratamiento.

5. Valorar la frecuencia de aparición de efectos adversos.

6. Identificar posible uso inadecuado de estos medicamentos.

\section{Materiales y métodos}

\subsection{Tipo de estudio}

Estudio epidemiológico descriptivo transversal.

\subsection{Población}

Se incluyeron pacientes que están en tratamiento con al menos un principio activo del grupo N05 y libremente decidieron participar en el estudio, independientemente de su indicación. Se seleccionaron en la Oficina de Farmacia, en el momento de la dispensación del medicamento. Respondieron al cuestionario un total de 115 personas.

\subsection{Recogida de datos}

Se hizo mediante la aplicación de un cuestionario (Anexo) formado por 15 preguntas cerradas, previa revisión bibliográfica (Lorenzo et al., 2009; Flórez et al., 2014; Goodman, Gilman, 2007; WHO, 2019). Para elaborar el cuestionario se efectuó una búsqueda de los principios activos y de sus correspondientes especialidades farmacéuticas comercializadas. Las fichas técnicas (AEMPS, 2019) de cada una de ellas fueron consultadas para poder elaborar algunas respuestas del cuestionario. 


\subsection{Variables de estudio}

3.4.1. Variables epidemiológicas de persona

- Sexo

- Edad

- Nivel de estudios

- Profesión

- Situación laboral

\subsubsection{Variables dependientes}

- Benzodiazepina utilizada

- Origen de la prescripción (Atención Primaria o especializada)

- Indicación

- Dosis

- Condición de uso (Tratamiento Agudo o Crónico)

- Duración del tratamiento

- Efectos adversos

\subsection{Estudio piloto}

Se realizó aplicando el cuestionario a 17 personas y sirvió para elaborar el cuestionario definitivo.

\subsection{Aplicación del cuestionario}

Mediante entrevista, realizada por el propio investigador, durante febrero y marzo de 2019.

\subsection{Análisis de los resultados}

Se elaboró un formulario de Google con su correspondiente hoja de cálculo como base de datos que posteriormente se exportó a Microsoft Excel, a partir de la cual se calcularon las frecuencias y porcentajes para crear las correspondientes tablas y figuras. Las diferencias entre variables se estudiaron mediante el estadístico chi cuadrado y el nivel de significación $\rho$. 


\section{Resultados y discusión}

\subsection{Características generales de la muestra}

Del total de personas incluidas en el estudio, el $27,8 \%$ son hombres y el 72,2 $\%$ mujeres (Tabla 1).

Atendiendo a la edad, un 40,0 \% tienen 70 o más años, seguidos de los que tienen entre $50-59$ con un $22,6 \%$. El grupo minoritario son los de edad entre 30-39 con un 4,4\% (Figura 1).

El nivel de estudios de casi la mitad (49,6\%) son estudios primarios, el 18,3 $\%$ bachiller o ciclo formativo superior, el 18,3\% estudios superiores y el 13,9\% estudios secundarios (Figura 2).

En cuanto a su situación laboral, el 49,6 \% de los pacientes son jubilados, el $20,0 \%$ tienen contrato indefinido, el $12,0 \%$ son amas de casa y el 7,0 \% están desempleados (Figura 3).

\subsection{Valoración del uso de benzodiazepinas}

\subsubsection{Uso de principios activos}

El principio activo más consumido es Alprazolam (36,5\%), seguido de Lorazepam $(29,6 \%)$ a diferencia del estudio de consumo entre los años 2000 y 2012 (AEMPS, 2014) donde el más consumido, como ansiolítico, era el Lorazepam seguido de Alprazolam. El menos consumido es Ketazolam (0,9 \%) (Figura 4).

El consumo de Alprazolam es mayoritario en hombres (53,1 \%); Bromazepam, Diazepam y Lormetazepam tienen uso similar en ambos sexos, y Lorazepam es utilizado principalmente por mujeres (34,9\%). Diferencias significativas $\rho=0,048$.

Las personas entre 30-39 años, más jóvenes, utilizan mayoritariamente Alprazolam $(60,0 \%)$ y Diazepam (40,0 \%). En cambio, las personas mayores, con $70 \mathrm{o}$ más años, usan preferentemente Lorazepam (39,1\%) seguido de Alprazolam (30,4 $\%)$. Al contrario ocurre a las personas entre 60-69 que toman preferentemente Alprazolam (42,1\%) seguido de Lorazepam (31,6 \%).

Un 33,3\% de las personas con trabajo temporal utilizan Alprazolam, el mismo porcentaje que consume Diazepam. Un $34,9 \%$ de los trabajadores indefinidos consumen Alprazolam, seguido de un 30,4\% que toma Lorazepam. Los estudiantes utlizan solo Alprazolam y Bromazepam. 


\subsubsection{Cómo son utilizadas las benzodiazepinas}

Un 96,5\% afirma consumir el fármaco bajo prescripción médica, mientras que un 3,5\% lo hace sin ella. La mayoría de las veces $(66,7 \%)$ el tratamiento es prescrito en Atención Primaria, mientras que el 27,9 \% en Psiquiatría y el porcentaje restante en otras especialidades.

Esto sucede así tanto en hombres $(80,0 \%)$ como en mujeres $(61,7 \%)$ y en los distintos grupos de edad, excepto las personas entre 40-49 años, donde al 53,9 $\%$ le prescribe el psiquiatra.

En cuanto a la indicación, acorde con estudios previos (García et al., 2004), en la mayoría de los casos (51,3\%) se prescribe el medicamento como ansiolítico, el $40,9 \%$ como hipnótico y el 7,8 \% como relajante muscular. Nadie en este estudio utilizaba el fármaco como anticonvulsivante.

Tanto en hombres $(50,0 \%)$ como en mujeres $(51,8 \%)$ se prescribe principalmente como ansiolítico. Como relajante muscular se prescribe más a hombres $(18,8 \%)$ y como hipnótico a mujeres (44,6 \%). Diferencias significativas $\rho=0,02$.

$\mathrm{La}$ tendencia en todas las edades es prescribir el fármaco principalmente como ansiolítico, salvo en las personas con 70 o más años, que el 60,9 \% lo utilizan como hipnótico.

A los 3 estudiantes se les prescribe como ansiolítico. A diferencia de otros estudios (Simó, 2012), el 50,9 \% de los jubilados lo utilizan como hipnótico, mientras que al $62,5 \%$ de los desempleados y al 47,4 \% de los jubilados se les receta como ansiolítico. Diferencias significativas $\rho=0,005$.

La mayoría de pacientes $(72,2 \%)$ toman siempre la dosis prescrita, pero el 19,1 $\%$ toman la dosis que cree conveniente por ser un tratamiento de larga duración y conocer bien los efectos del mismo.

Se observa el mismo comportamiento en hombres y mujeres y atendiendo a la situación laboral.

El 87,0 \% de los encuestados tienen un tratamiento crónico (más de un mes), mientras que para el 13,04\% el tratamiento es agudo. Concretando, el 60,9\% lleva en tratamiento más de 2 años, mientras que solo el 13,0\% lleva menos de un mes.

Atendiendo al sexo, la mayoría de hombres $(53,1 \%)$ y de mujeres $(63,9 \%)$ están consumiendo benzodiazepinas desde hace más de 2 años. Solo un 25,0\% de los hombres y un $8,4 \%$ de las mujeres llevan menos de un mes en tratamiento.

El 66,7 \% de las personas entre 18-29 años, como es de esperar, llevan consumiendo el fármaco menos de un mes. Por el contrario, como señalan otros autores (Simó, 2012), el 87,0 \% de las personas con 70 o más años llevan tomando benzodiazepinas más de 2 años. Diferencias significativas $\rho=0,00001$.

En cuanto a la situación laboral, el 66,7 \% de los estudiantes tienen un tratamiento agudo (menos de un mes), mientras que el $80,7 \%$ de los jubilados y el 64,3 
$\%$ de las amas de casa tiene un tratamiento crónico (más de dos años). Diferencias significativas $\rho=0,005$.

El 73,9\% de los encuestados afirman tomar siempre la misma dosis, mientras que el $26,1 \%$ no lo hace.

Llama la atención que casi la mitad $(46,1 \%)$ no ha tenido ningún efecto adverso. Los más frecuentes son tolerancia y dependencia (34,8 \%) y los menos frecuentes somnolencia y sedación excesiva (Figura 5).

En mujeres el efecto adverso más frecuente es dependencia $(37,4 \%)$ y en hombres ataxia, disartria o amnesia (12,5\%), aunque la mayoría de hombres y mujeres afirma no tener ninguno.

Por edad, las personas mayores, 70 o más años, presentan principalmente dependencia y tolerancia (43,5\%). Un 23,1 \% de las personas entre 40-49 años afirman tener 2 o más efectos adversos.

Los jubilados afirman a partes iguales $(40,4 \%)$ no tener efectos adversos y sufrir dependencia y tolerancia. Destaca que un 37,5\% de los desempleados afirman tener 2 o más efectos adversos.

Teniendo en cuenta que la duración de tratamiento recomendada es de 1 mes, un $83,7 \%$ de las personas afirman que no han dejado de tomar el fármaco trascurridos 6 meses porque siguen la posología indicada por el médico. Un 12,0 \% afirman que intentaron dejarlo, pero tuvieron efectos negativos y siguieron tomándolo.

\section{Conclusiones}

1. El perfil de los pacientes en tratamiento con benzodiazepinas corresponde a mujer de 70 o más años, con estudios primarios y actualmente jubilada.

2. Los principios activos más consumidos son Alprazolam y Lorazepam y el menos utilizado Ketazolam.

3. Los hombres consumen principalmente Alprazolam y las mujeres Lorazepam y el consumo aumenta con la edad, siendo el grupo de personas con 70 o más años el que más benzodiazepinas consume.

4. Las benzodiazepinas se prescriben mayoritariamente en Atención Primaria y dentro de la Atención Especializada en Psiquiatría.

5. Se prescriben principalmente como ansiolíticos, tanto en hombres como en mujeres y en todas las edades, excepto en personas de 70 o más años que se utilizan como hipnóticos.

6. La mayoría de las personas en tratamiento toma siempre la misma dosis.

7. Los tratamientos son principalmente crónicos (más de 2 años) y solo una pequeña parte agudos (menos de un mes). Además, las personas más jóvenes, entre 18-29 años, son las que tienen tratamientos más cortos y las personas mayores, 70 o más años, tratamientos más largos. 
8. Los efectos adversos más frecuentes son dependencia y tolerancia $(34,8 \%)$ y los menos frecuentes somnolencia y sedación excesiva (3,5\%), aunque casi la mitad de los encuestados (46,1 \%) afirman no sufrir ninguno.

9. Por tanto, según lo expuesto, se podría mejorar el uso de las benzodiazepinas, principalmente la duración del tratamiento ajustándolo al mes que indican las Fichas Técnicas, implementando también medidas sociales adecuadas a las necesidades de los pacientes.

\section{Bibliografía}

AEMPS: Agencia española de Medicamentos y Productos Sanitarios [Internet]. Madrid: AEMPS; c2013 [citado el 16 de febrero 2019] Centro de Información Online de Medicamentos; Fichas Técnicas. Disponible en: https://cima.aemps.es/cima/publico/home.html

Agencia española del Medicamento y Productos Sanitarios. Observatorio del Uso de Medicamentos. Utilización de Medicamentos Ansiolíticos e Hipnóticos en España Durante el Periodo 2000-2012. [Internet]. Madrid: AEMPS; 2014 [actualizado 27 enero 2014; citado 16 febrero 2019]. Disponible en: https://www.aemps.gob.es/medicamentosUsoHumonao/observatorio/docs/ansiolíticos_hipnoticos-20002012.pdf

Flórez J et al. Farmacología Humana. 6th ed. Barcelona: Masson S.A.; 2014.

García del Pozo J et al. Utilización de ansiolíticos e hipnóticos en España (1995-2002). Rev. Esp. Salud Publica 2004; 78(3):379-387.

Goodman LS, Gilman A. Las bases farmacológicas de la terapéutica. 11th ed. Madrid. Internacional: McGraw-Hill; 2007.

Hollingworth SA, Siskind DJ. Anxiolytic, hypnotic and sedative medication use in Australia. Pharmacoepidemiol Drug Saf. 2010; 19(3):280-288.

Khong TP et al. Potential impact of benzodiazepine use on the rate of hip fractures in five large European countries and the United States Calcif Tissue Int. 2012; 91(1):24-31.

Lagnaoui $\mathrm{R}$ et al. Patterns and correlates of benzodiazepine use in the French general population. Eur J Clin Pharmacol. 2004; 60(7):523-529.

Lorenzo P et al. Farmacología Básica y Clínica. 18th ed. Madrid: Médica Panamericana; 2009.

Ministerio de Sanidad, Servicios Sociales e Igualdad. Secretaría de Estado de Servicios Sociales e Igualdad. Delegación del Gobierno para el Plan Nacional sobre Droga. Encuesta sobre alcohol y drogas en población general en España: EDADES 2011-2012. 2013. Disponible en: http://www.pnsd.msc.es/Categoria2/observa/pdf/EDADES2011.pdf

Rayón P et al. Benzodiazepines consumption in Spain. Eur J Clin Pharmacol 1997; 52:321-323.

Simó Miñana J. Utilización de medicamentos en España y en Europa. Aten Primaria. 2012; 44(6):335-247.

WHO Collaborating Centre for Drug Statistics Methodology [Internet]. Oslo: WHO; c2009 [actualizado 13 enero 2019; citado 16 de febrero 2019] ATC/DDD Index. Disponible en: https://www.whocc.no/atc_ddd_index/ 